\title{
COVID -19 and Orthopedic Surgery: Strategies from Freeze to the Light at the End of the Tunnel
}

\author{
Sunil S Nikose ${ }^{1 *}$, Devashree Nikose ${ }^{2}$, Shashank Jain ${ }^{3}$, Kiran Saoji ${ }^{4}$, Aditya Kekatpure ${ }^{5}$ and Sandeep \\ Shrivastava ${ }^{6}$
}

${ }^{1}$ Department of Orthopaedic surgery and Director of Centre of simulation studies, Jawaharlal Nehru Medical College, India

${ }^{2}$ Medical Doctor, NKP Salve Institute of Medical Sciences, India

${ }^{3}$ Department of Orthopedic surgery, Jawaharlal Nehru Medical College, India

${ }^{4}$ Department of Orthopedic surgery, Jawaharlal Nehru Medical College, India

${ }^{5}$ Department of Orthopedic surgery, Jawaharlal Nehru Medical College, India

${ }^{6}$ Department of orthopedics, Jawaharlal Nehru Medical College, India.

*Corresponding author: Sunil S Nikose, Department of Orthopaedic surgery and Director of Centre of simulation studies, Jawaharlal Nehru Medical College,

\begin{abstract}
The Corona virus SARS-CoV-2 (COVID-19) crisis has rapidly spread worldwide after the emergence of the pandemic in Wuhan in December 2019 it has resulted in health, economic and other non-health impacts with the healthcare systems of developed and developing countries being overwhelmed throughout the globe. It has also impacted the surgical branches and orthopedic surgery worldwide, more so the elective orthopedic surgery causing much apprehension, emotional instability and continued disability in the geriatric population who are waiting for the surgery. While this is true, healthcare professionals from all branches especially the critical care, intensivists, and physicians in high dependency units have been overwhelmed and the resources depleting fast due to stretch by this pandemic. The elective surgeries bear the brunt of the pandemic, with emergency orthopedic surgery and trauma demonstrating an increase after the lockdown has been eased and traffic has resumed on the road. Most orthopedic associations across the globe were quick to issue safety guidelines for healthcare professionals and orthopedic surgeons, including the Indian Orthopedic Association (IOA), the British Orthopedic Association (BOA) and the American Academy of Orthopedic Surgery (AAOS). Teaching and research activities are also being hampered due to these unprecedented times. As we restart elective surgical procedures during and post-COVID-19, it is obligatory to apprehend what techniques and surgical etiquette need modification or enforced to reduce the risk of disease transmission throughout the severe acute respiratory syndrome (SARS)-CoV-2 pandemic. This review focuses on COVID 19 effect on orthopedic surgery before, during and after COVID 19
\end{abstract}

Keywords: Corona Virus; COVID-19; SARS-CoV-2; orthopedic surgeons; Pandemic

Abbreviations: COVID-19-Corona Virus Disease 2019; IOA-Indian Orthopaedic Association; BOA-British Orthopaedic Association; AAOS-American Academy of Orthopaedic Surgeons; CDC-Centre for disease control and prevention, USA; IT-Information technology; AI-Artificial intelligence; ACSAmerican College of surgeons; WHO-World Health Organization; SARS-Severe Acute Respiratory Syndrome -related coronavirus; MERS-Middle East Respiratory Syndrome - related coronavirus; HDU-High dependency Unit; OR-Operating room; ICU-Intensive Care Unit; MVA-Motor Vehicular Accident; PPE-Personal Protective Equipment; AIOT-Alliance of International Organizations of Orthopaedics and Traumatology AOA-Australian Orthopedic Association; LOS-Length of stay; PPE-Personal protective equipment; NICE- National Institute for Health and Care Excellence; NHSNational health Scheme (United Kingdom) CT- Computed tomography; ADL- activity of daily living 


\section{Introduction}

Corona virus is a positive-stranded RNA virus of 0.06-0.14 microns diameter having a crown like appearance, due to spike like glycoproteins on envelope and is sensitive to ethanol, chloroform, chlorine containing disinfectant, ether and peracetic acid. The COVID stands for coronavirus disease and was revealed in December 2019 at Wuhan city of China resulting in a worldwide acceptable abbreviation of the disease as COVID-19. This pandemic of COVID-19 presently is escalating throughout the world at a scaring proportion causing increases infectivity and mortality in contrast with the severe acute respiratory syndrome (SARS) or Middle East respiratory syndrome coronavirus (MERS-CoV) [1]. Within weeks, it had resulted in a global standstill with lockdowns of variable durations across the world and a number of flare-ups were identified in Italy, Spain, France, and the USA followed by the World Health Organization (WHO) announcing the Coronavirus disease 2019 (COVID-19) as a pandemic on the 11th March 2020, when more than 100,000 (105) people were infected among the hundred countries worldwide [2]. It has been almost more than five months since the outbreak and it seems that this virus is highly contagious and less likely to disappear from our lives despite a fast-track vaccine production in the very near future which has conflicting claims of partial effectiveness or safety of around 50\%$60 \%$ suggesting that the public health measures would be largely needed to keep this unprecedented pandemic well within control. The lockdown measures which were imposed in several countries to keep the virus at bay have devastated the economy greater than the "Great Depression" and exposed a crippled health system not tuned to the health of the individuals.

In most scenarios, the orthopedic fraternity are being redeployed for treating the critical respiratory illnesses requiring ventilatory support and non-critical or asymptomatic COVID-19 patients. However, how the Coronavirus might affect the musculoskeletal system, bone and joints of an individual has not been described and there is an almost no information related to this for a specialty who are used to deal with trauma, accidents as their emergency duties are concerned. There seems to be a demand for mandatory preparation of the orthopedic community regarding understanding the pathology, respiratory manifestations and respiratory complications of the disease along with mechanical ventilation management so that they would be better tuned to manage these patients on ventilatory support when their critical care or Intensive care unit (ICU) colleagues appeal a helping hand to at least relieve their temporary burden and stress. Performing orthopedic and trauma surgical procedures have become notable obstacles due to the limited accessibility of the hospital workforce and surgical installations which have been diverted for the control of COVID -19 disease. However, the orthopedic diseases which have been back-seated results in considerable pain, limited activity and incapacitation to a patient suffering from a chronic musculoskeletal illness and who becomes incapable of performing activities of daily living (ADL) especially pertaining to ambulation and dexterity [3]. Moreover, there have been profound detrimental consequences on the manufacturers of orthopedic implants, devices and instruments along with a complete standstill to the ongoing orthopedic research, conferences, academic activities, and further physician career development activities [4]. A lion's share of individuals who stand in need of elective orthopedic procedures are geriatric with the likelihood of requirement for an Intensive care unit (ICU) support during their recovery or postoperative care pathway. The complete lockdown previously might have substantially reduced the number of motor vehicular accidents (MVA) during the past few months, but it had by no means substantiated reduction in the requirement of an emergency orthopedic procedure. We can theoretically predict that most or all practices related to elective orthopedics will be delayed or postponed or abandoned resulting in an anticipated surge of patients in the coming months with delayed treatment which would correspond with significantly increased morbidity until this subset of patients receive their treatment.

The coronavirus is spreading tremendously throughout the world and the August month statistic reveals that India is the new epicenter of the infection resulting in frequent swirling of the cluster of patients in different parts of the country despite stringent lockdown measures from 25th March, 2020 to 31st May 2020 along with unlocking phases from June onwards to revive the economic activities and livelihood of people. In the current situation, most governments, as well as the medical associations, are unable to measure the precise position of COVID-19 in a given population and the guidelines offered by them are frequently changing resulting in chaos and confusion across the medical fraternity. Therefore, considering these fluctuating guidelines and the variable behavior of coronavirus the elective orthopedic surgery performance needs a thorough work-up of COVID-19 to reduce the risk to the patient, and caregivers including operation room (OR) personnel, ward staff, residents, and faculties. It is widely accepted with various mathematical calculations that the frequency of COVID-19 individuals must diminish for a fortnight before resuming any elective orthopedic procedure [5]. Numerous countries have been able to control and will probably soon achieve this target in Europe by a variety of means. While the virus-free crisis is thought to be deemed under control, it should be taken into consideration that the pandemic is not yet over and frequent clusters along with a second larger and more potent strain of infections could lead to the premature and sudden lifting of interventions related to the medical and surgical facilities [6]. To ensure that the patient and medical personnel remain safe the protection has to be blanketed involving all the contacts, including tracing and testing, isolation of even asymptomatic individuals and thereby reducing the number of infections. This is largely possible due to sustainable and regulatory initiatives within healthcare and governmental administrative infrastructure along with following of self-discipline within the 
population such as avoidance of overcrowding, social and physical distancing, and proper wearing of masks in the future. Healthcare administrators consisting of all the specialties and subspecialties need to design a roadmap for the resumption of the safe pathway of care for any elective surgery. Nonetheless, despite more than a few millions of literatures in the past five months no relevant literature has been available till date regarding the establishment of an ambulatory facility, resumption of safe elective surgical procedures or related activities [7]. The WHO is also constantly updating its bibliographic databases, hand searching, expert committee advice, and expert-referred scientific articles regarding COVID-19 on a day to day basis from Monday to Friday. As the number of patients continues to rise exponentially throughout the world this article would aim to highlight the challenges faced by most of the medical professionals along with the orthopedic and trauma surgeons including academicians, along with the optimal care of patients during and in the long term after the pandemic, effects on the academic activities and research activities, short - term and long term recommendations. The present article is also helpful to other subspecialties with minor relevant modifications.

\section{Orthopaedic Associations Recommendations and Guidelines During COVID-19}

Most orthopedic associations across various countries were very quick to issue safety guidelines for healthcare professionals and orthopedic fraternities, including the Indian Orthopaedic Association (IOA), the British Orthopaedic Association (BOA), the American College of Surgeons (ACS) and the American Academy of Orthopaedic Surgery (AAOS). As per the Indian Orthopaedic Association's advisory and guidelines for orthopedic practice during COVID 19 pandemic updated on 8th June 2020, the susceptible population was color-coded and graded as low risk (green), medium risk (orange), high risk (red), and very high risk (black) depending on the associated co-morbid conditions, age of the individuals and immune status of individuals. The advisory suggested few modifications in orthopedic outpatient and inpatient admission practices to contribute to the healthcare needs of the country, anticipating an increase in ventilator beds along with the rational use of the resources. Patients triaging for surgery were in red for absolute indications, yellow for relative indications, and green for non-operative management of patients. The elective surgical pathway for future were categorized into a "green pathway" (COVID free) for elective cases and "blue pathway" (COVID care) for COVID positive or clinically suspected patients. Under these guidelines, it is recommended that elective surgeries for COVID free patients should be performed in a separate hospital or separate dedicated arena in a huge hospital setup with independent entry and exit. Hospital personnel should be screened regularly and sampled for COVID-19 while protecting both the green and blue pathways in a separate dedicated environment for the tasks assigned. The blue pathway allows only urgent and emergency orthopedic procedures to be performed along with the care of these patients for COVID related illnesses. It also recommended the use of personal protective equipments (PPEs) as advised along with special donning and doffing training for COVID 19 as per the requisite guidelines [8].

The British Orthopaedic Association (BOA) guidelines are being continuously revised based on the reflection of the disease, and the latest document, updated version 7 updated on 27 July 2020 with a strapline of "Caring of patients, supporting surgeons" provides core principles regarding planned care during hospitalization and diagnostic services and about restarting of aspects of elective orthopedic care that has been postponed or suspended due to COVID-19. The BOA along with the National Institute for Health and Care Excellence (NICE) recommendations comprises mainly three sections namely 1) Prioritising patients for surgery, 2) Infection prevention and control measures for planned surgery and 3) Capacity issues for resuming surgery which includes a continuing care pathway starting at the before care (Preoperative), prevention and control, guidelines during care and ends with the ongoing care of the patient $[9,10]$. These guidelines are based on literature available till date along with advice from various specialists working with the National Health Scheme (NHS) and are comprehensive and exhaustive and comprise of all stakeholders including healthcare professionals and commissioners, while the academic activity of BOA congress 2020 during 15 to 18 September will be held in a virtual mode consisting of pre-recorded webinars and live sessions [9].

The Alliance of International Organizations of Orthopaedics and Traumatology (AIOT) released a joint statement about COVID 19 best practices on 28 April 2020 and recommended a collaboration leading to worldwide research opportunities and maximising the existing hospitals, institute networks and efficiency during the pandemic leading to determination of negative impacts of the COVID 19 related to orthopaedic and trauma care worldwide. The general consensus was to cater to the safety of patients and treating staff, update knowledge regarding new evolving guidelines, rotate teams, prepare emergency contingency planners, rationalise use of PPE and be prepared for shortages of medical supplies. While performing emergency and urgent procedures, they have recommended that a surgical review committee should prioritise and schedule each patient and the operating room should be secluded away from high patient traffic areas, with taping of anteroom and the use of negative pressure air or turning off the positive pressure air for fear of the spread of coronavirus. While there are several other recommendations of AIOT apart from those described above certain important recommendations are to postponement of all orthopaedic elective surgeries along with scheduling only urgent and emergency patients, telemedicine facilities development for health, diagnostic and rehabilitative purposes, engaging the use of internet and technology for trainee teaching and academics, screen patients and personnel attending 
outpatient and in-patient facilities for COVID-19 and to implement a surgical review committee to assist in surgical cases prioritization along with a phasic return to the elective orthopaedic list [11]. The recommendations were exhaustive and comprehensive, and the strength and quality of recommendations were based on 83 research articles regarding the guidelines and each was assessed using a grading system regarding reliability index as good, fair or poor.

Likewise, Australian Orthopaedic Association (AOA) and Australian Orthopaedic Trauma Society (AOTS) modified their statements regarding a safe approach to return to elective surgery on 24 April 2020 whereby the healthcare facilities should "choose wisely" until the usual pre pandemic era is established. They strongly recommended that urgent and essential orthopaedic surgery needs of Australians must be preserved at its present level during the COVID-19 pandemic and since the supply chain and logistics are mostly off-shore their consumption should be judiciously and wisely employed along with a rationed system while avoiding double operating rooms, consultants performed surgeries based on experiences, and a separate category named as category 3 wherein the prioritization of the patients should be allotted towards " high value and low risks procedures" so that the ICU beds are spared for the needy COVID 19. Non-acute and lesser preference procedures must be adjourned in all hospitals until such time as the consequences regarding the COVID-19 pandemic on the Australian health organization are fully known with the ultimate responsibility placed on the orthopaedic teams caring for the patient using safe and sound professional judgement [12]. In the present literature survey, we found more or less similar recommendations by almost all the orthopaedic associations of the world.

The Centre for Disease Control (CDC) and Prevention has been updating the guidelines regarding clinical practices during COVID 19 and the latest revised one was provided on 06 August 2020. These guidelines includes clinical care guidance, therapeutic options to consider, guidance for home care and care of special need patients like children, infants and neonates, geriatric population, pregnant and breast feeding women It includes training of the staff and preparation of the clinic before the patient arrive in a manner that the surgeon should have knowledge regarding patients at higher risks, planning for telemedicine appointments and consultations as well as rescheduling non-urgent appointments, staying connected with the local health department pertaining to newer guidelines regarding the spread in the local community and effective signage at the entrance and waiting areas to educate the community regarding prevention of disease like wearing of masks, social distancing and upkeep and disinfection of home. While the waiting area should be clear of all hindrances, it should be sterile, having barriers, distancing methods with plenty of supplies of soap, tissues, alcohol-based hand rubs and trash cans. Once the patient arrives, the sick and non-sick patients should be segregated along with notification to the local health department if symptoms of coronavirus disease are suspected [13].

\section{Orthopaedic Practice During COVID 19}

While there is a significant effect of the COVID-19 in an unimaginable way throughout the world concerning the health of society, it has also caused an unprecedented crisis in orthopedic practice and this effect is likely to last in the near future for the uncertain duration of time. During COVID 19 clinical practice has been reshaped, substantially altered and affected by three major objectives such as: 1) Surgeries can be performed in case of clinical emergency; 2) Health care professionals (HCPs) and patient protection; and 3) Conservation and judicious uses of health-care assets. There is an inherent requirement of triaging the patients during the COVID-19 crisis and reducing unnecessary admission associated with minor trauma as well as other disorders. Also, there is a need to avoid scheduling non-urgent and physical appointments unnecessarily to the great extent for routine evaluations and trivial problems while ensuring that the quality of care is optimal and high quality [14]. The advent of such a crisis offers a timely potential platform for reflecting and analysing onthe-job usage of novel technologies.

Accordingly, elective, non - emergent procedures which require a greater stay in hospital than 23 hours may be delayed or cancelled, but day care surgeries can be carried out with the patient being kept on the list early in the morning like arthroscopies and other day care procedures. This will have a particular influence on arthroplasty of the hip and knee, spinal deformity corrections, and paediatric elective surgical procedures. Patients who undergo such procedures typically need longer hospitalizations of more than three to five days and these patients may require a postoperative ICU care and stay due to complications posing an additional threat to an already burdened and incapacitated health system along with increase in the risk of nosocomial infections [15]. For these reasons, a professional and judicious approach is to be used for non - emergent elective procedures requiring more resources and a longer duration of hospital stay. However, this might result in unintentional adverse effects on patient health and care, especially in patients experiencing worsening pain from degenerative joint diseases, spine conditions and neurological compromise. A wise approach to these patients would be to consider temporizing painalleviating measures in their treatment, wherever possible like intraarticular corticosteroids or simpler nerve root blocks, facet block and other less invasive tailored therapeutic modalities [16].

Special care must be exercised whenever emergency procedures are needed such as trauma, injuries, fractures, and malignancy where postponement is extremely difficult, hence, wearing of PPEs as per the proper guidelines of donning and doffing must be employed to prevent the surgical team from becoming morbid. A combination of interventions like wearing a N95 respirator mask (filtering face piece respirators or FFRs), face shield, gloves, medical 
gowns and PPEs has been of proven value and is recommended for protection of the surgical team by excluding extremely minute viral organisms [17]. The rapid spread of this disease has led to weakening of most international markets with significant economic loss and unavailability of commodities and raw material in time with interruption of the global supply chain causing compromised situation and risking the lives of healthcare workers [18].

\section{Academic Activities During COVID-19}

On the academic front, almost all medical institutions classroom teaching has ceased, and large numbers of medical student examinations are either postponed or cancelled in wake of COVID-19 spread. Student teaching, evaluation and quality control are more or less online and is much difficult to achieve without violating privacy policy and subsequently the formative assessments are also influenced accordingly. Most of the instructor led teachings are conducted via webinars, online modes or e-learning resulting in less face-to face interaction, negligible feedback from the students, and lack of accreditation, requiring intense self-discipline causing excitement for few tech-savvy teachers and headache for few others who are not used to technology thereby causing the learning process much more difficult. The training regarding clinical and surgical skills of the residents is jeopardised with only limited exposures in a simulation-based set-up which is demanding and less reproducible in clinical practice. The major hurdles in online teaching are lack of good internet connectivity, access to devices, digital illiteracy, attention span, poor audio-video devices, and multi-tasking is a hindrance which nevertheless can be partly solved using an excellent Information Technology (IT) department in a medical school. The overload of online resources and webinars are in itself described as some as a pandemic situation of e-learning but this distance education is here to stay even post COVID-19 and the orthopaedic surgeons would require cutting-edge technology, use of artificial intelligence (AI) for the training and educational needs of graduate and post graduate students while converting the "crisis into opportunities" and keeping a positive attitude during this time and overcome these challenges by motivating younger individuals.

The Ministry of health and family welfare of India issued an advisory for hospitals and medical institutions and recommended that all educational institutions and examination processes be conducted via electronic communication so as to reduce anxiety amongst students, teachers and parents alike. Institutions are also asked to notify help-line numbers / emails that students will be able to access for their queries [19]. Till date undergraduate medical schools across India have not yet started and further delays are likely until an uncertain period of time.

\section{Care During Surgery in the Present Scenario}

The likelihood of getting infected in the operating room (OR) is very high and considering the long training period and learning curve of a surgeon the replacement of a surgeon is not a good option.
Hence, all the procedures must minimize viral dissemination during the procedure and includes only essential workforce gain access to the operating room (OR), ideally having a negative pressure OR, a two separate "buffer zones" along with another absolutely "clean zone" along with PPEs donning and doffing the PPEs in separate buffer zones. There should be two separate ante rooms for induction of patients and scrubbing of patients which are sealed by interlocking doors apart from airdrop sealing. Only the most senior of operating surgeons should be present during surgery. Patients should also have surgical masks and caps. The outline of safety practices along with proposed modification of the operating room (OR) is depicted in the (Figure 1).

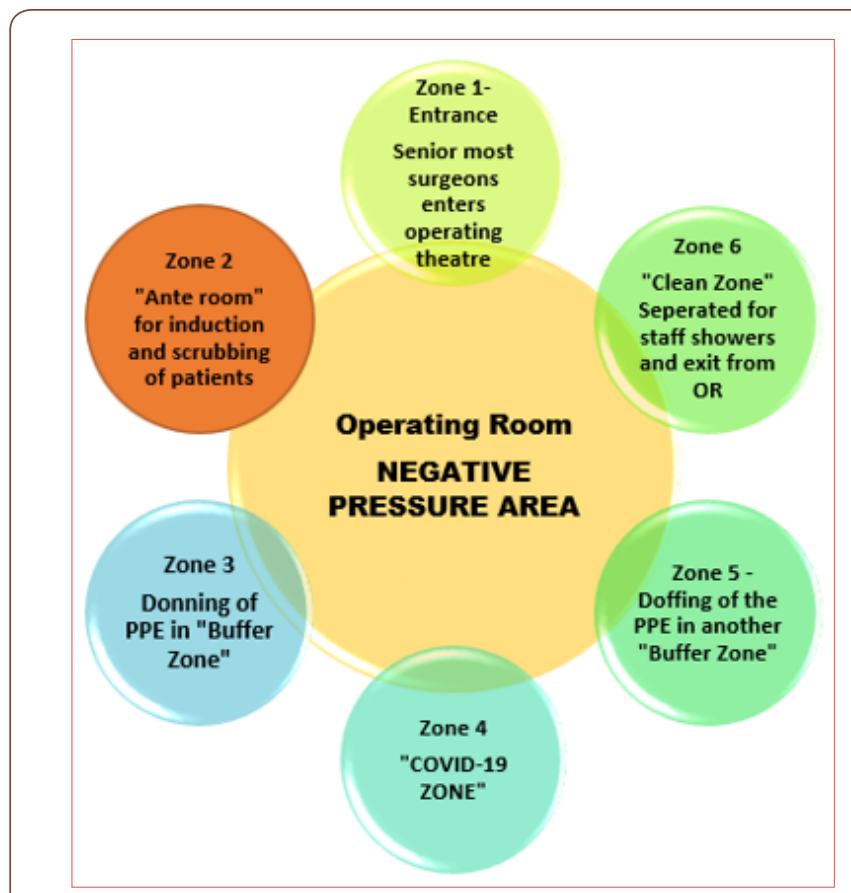

Figure 1: Operating Room (OR) modifications and safe practices during COVID-19 period.

It is advised to run operation theatres with negative pressure ventilation facilities. Moreover, it is of utmost importance to reduce the number of personnel at the operating theatre along with establishment of a committed squad consisting of an experienced surgeon, a pair of residents who are appropriate to deal with every situation. During the surgical procedure, the assistant, a dedicated nurse or a paramedical staff member may be assigned where needed. The unit will organize the monitoring and working on suspicious or positive COVID-19 patients, and willingness to be stay aside secluded from others in the department to minimize the possibility of cross-infection along with rotating interchangeable teams to be set up for replacement of team that is accidentally exposed to an infected patient with a heavy viral load [20]. Notably, asymptomatic COVID-19 infection may also occur in elective patients which has a capacity of increasing the patient's mortality. Likewise, the emergency patients might have existing COVID-19 disease which increases their risk of mortality due to general anaesthesia. Therefore, non-operative management over surgical 
intervention should always be favoured where we need to give each patient outstanding quality medical care rather than deny them in fear of being contaminated with COVID-19.

\section{Resource Management During COVID-19}

Most orthopedic facilities were converted to COVID-19 hospitals and the clinics, operating rooms along with high-dependency units (HDU), were assigned to treat COVID-19 individuals. Even as the figure of individuals requiring mechanical ventilation surges, a substantial rise in ventilated beds are required to be available to cope up with both pre COVID-19 occurrence heights even as new COVID-19 patients are on the rise. The availability of blood transfusions is hypercritical in inclusion to the scarcity of ICU spaces. This has a probability to restrain the dimensions for conducting the majority of difficult and formidable situations like revision treatments including infections of prosthetic joints, reconstructive surgeries and staged surgeries wherein the first stage of surgery had been finished in the Pre COVID 19 era. Also, consideration should be given to the extended time duration needed for imaging modalities, because scanners need careful cleaning and disinfection between two patients. Therefore, the service delivery would pile up when confronted with the rise in requests leading to many obstacles, accompanied by a rise in delay of care, a thing that should be distinctly conveyed to all the patients. Healthcare facilities operating with bottle neck pricing may discover that the costs are increasing well beyond those formerly foreseen when these were at first transacted, thereby threatening many service providers economic survival $[20,21]$. They will need to ensure preparedness along with tolerance to a potential subsequent wave increase in COVID-19 - related sickness as they plan to reopen elective services at times when social distancing steps start to be loosened up.

\section{Orthopaedic Associations Guidelines Post COVID-19}

After the COVID-19 pandemic began, almost all surgical services had recommended to postpone the elective orthopaedic surgical procedures, such as joint replacement surgery, arthroscopies, reconstructions and spine surgeries. Presently, a group of experts has released guidelines to direct the secure restart of elective orthopaedic surgery and these recommendations appear in The Journal of Bone and Joint Surgery, July 15, 2020 edition [20]. In the post-outbreak phase, orthopaedic surgeons will choose appropriate personal protective equipment ( PPE) based on Level II requirements, including a hair net, medical gloves, insulation gear, medical respirator (filtering face device $[\mathrm{FFP}] / \mathrm{N}, \mathrm{R}$, or $\mathrm{P}$ ), eye shielding (goggles and/or visor), and shoe protect.

Any patients visiting the outpatient orthopaedic clinic will initially be queried regarding the aetiology of their trauma alongside any positive infective history. All patients are recommended to undergo regular blood checks or a computed tomography (CT) of the chest if deemed necessary. There are three possible treatment schemes available according to the test results: (1) Patients with fever or abnormal regular blood tests should be moved to the fever clinic for more investigation.(2) Patients with good blood test results, CT tests and body temperature should go to the nucleic acid test transition ward during which a patient with negative test results can be admitted and (3) Patients with suspicious findings on CT examination should be transferred to the emergency department for COVID-19 nucleic acid and antibody testing.

If the test is positive, patients can be relocated to a delegated institution for COVID-19 treatment [20,21]. If the test is negative, they should go to the transitional ward for a second nucleic acid and antibody testing; if the test results remain negative, these patients can be admitted to the orthopaedic inpatient ward.

\section{Strategy for Resuming Elective Surgery}

Currently, it seems that patients in young age, lack of comorbidity and less invasive surgery like arthroscopies will have a very small chance of infection for the patient and surgeons alike. Thus, a plan for recommencement of elective surgery can be anticipated in four stages: (1) Less-invasive surgery for people less than 60 years of age with no comorbidity and a maximum hospital length of stay (LOS) of three days; (2) Surgery for all patients without comorbidity and a LOS of three days (3) Mini-invasive surgery for patients under the age of 60 , with co-morbidity or a LOS of more than three days, (4) surgery for all patients with comorbidity. In both of these steps, a thorough preoperative screening of patients would consider two scenarios. Firstly, patients who are by no means confirmed of COVID-19 or healed from the disease, should be isolated from patients with COVID-19 infection (suspected or confirmed) for at least a fortnight. And secondly, for the latter group the surgery should be postponed by at least eight weeks or until complete recovery.

\section{Pre-Operative Safeguards and Screen}

Patients should be monitored pre-operatively for instance through teleconference or videoconferencing and a thorough conversation should be arranged with the patient about his / her condition and involves questions relevant to COVID-19 such as contacts and exposure, signs and symptoms, and regarding any prior testing. Patients ought to consider every characteristic pertaining to their treatment that might alter owing to the present state of health emergency and be well informed of the restricted yet potential uncertainty of COVID-19 during the course of hospitalization and surgical treatment. Considering every precaution arranged by healthcare services that refrain from COVID-19 infections during the course of hospitalization, it is not possible to eliminate the possibility of infection 100 per cent [22]. Therefore, the surgical patient consent should be comprehensive beneath these expressions along with a more detailed screening in the preoperative phase. Unless, after preoperative examination, there is a suggestion or confirmation of COVID-19, the procedure may be postponed. 


\section{Possibility of a Second Wave Of COVID-19?}

Most of us are wondering about a possible COVID-19 second wave. Frequently the question arises, Isn't COVID-19's first wave enough or do we need to be concerned about a second wave? The response is yes because, considering the key factors, it's not only beyond the realm of imagination but something that is certainly possible. Indeed, second pandemic waves have already appeared in China and Japan. In December 2019, the SARS-CoV-2 virus first infected China, and the government imposed stringent restrictions in the Hubei region (epicentre of the infection) that could help manage the infection by March 2020.

If the state of present affairs are hitherto devastating, how significant are the influences with COVID-19 if a subsequent "second wave" of virus occurs? While the situation that lies ahead is not known, doubtful and difficult to forecast, there are sufficient historical instances to understand the magnitude of the pandemic's impact. For instance, the second wave was much worse than the first during the great influenza (Spanish flu) pandemic of 1918, due to a more virulent strain of the virus. Spanish flu accord an appropriate philosophy due to the complex genre and magnitude, and certainly both these infections cause severe respiratory illness and respiratory distress. Approximately 50 million people have died from the Spanish flu pandemic, much more than COVID-19 is causing $[22,23]$. It is likely that the coronavirus may do the same, a risk that we try to point out in this article because the governments are constantly promising that all will soon be fine, at least in countries that have already run the peak. What if they didn't? Are we prepared for the same? The COVID-19 second wave pandemic can have even more devastating effects.

\section{Conclusion}

The elective surgery is to bear the brunt of the pandemic and the emergency orthopedic surgery and trauma is demonstrating an increase after the lockdown has been eased and traffic has resumed on the road. Most orthopaedic associations across the world were quick to issue safety guidelines for healthcare professionals and orthopaedic surgeons. Though the COVID-19 hampered the services of orthopaedic surgeons and other surgeries as well, on the positive side, there are still some obvious benefits that the orthopaedic surgeons can derive from this calamity such as distressing themselves aside from the reduced exposure to radiation hazard, the chance to finalize incomplete works such as publications, thesis, dissertations and research, and most pertinently sparing quality time with their family members or beloved ones. While we are used to teamwork efficiencies as surgeons the teamwork is truly cardinal and each one should behave as a responsible team member or team leader of healthcare organization. We ought to be vigilant about protecting ourselves, our colleagues, our patients and our families. This is absolutely what the novel coronavirus wants to beat together. Although the current global outlook may not appear optimistic but with the development of herd immunity and a successful vaccine along with advanced preventive methodology we hope that COVID-19 will be eradicated and the new normal order can be restored.

\section{Funding}

None.

\section{Conflicts of Interest/Competing Interests}

The authors declare that they have no conflict of interest.

\section{Authors Contribution}

All the authors would like to express their great sense of appreciation to Miss Bhagyashree Nikose for her direct technical assistance during the planning and development of this review article at the peak of COVID - 19 pandemic including collection of data, arranging for daily reports of COVID - 19 from multiple websites and downloading the recommendations of various orthopedic associations across the globe. This was her voluntary "summer project".

\section{References}

1. Hassan SA, Sheikh FN, Jamal S, Esher JK, Akhtar A (2020) Coronavirus (COVID-19): A Review of Clinical Features, Diagnosis, and Treatment. Cureus 12(3): e7355.

2. WHO (2020) Coronavirus disease 2019 (COVID-19) situation report-51 19.

3. Bannock CD Unlike anything seen in peacetimee NHS prepares for surge in Covid-19 cases.

4. Haleem A, Javaid M, Vaishya R, Vaish A (2020) Effects of COVID-19 pandemic in the field of orthopaedics. J Clin Ortho Trauma 11(3): 498499.

5. Am Coll Surg (2020) Joint statement: roadmap for resuming elective surgery after COVID-19 pandemic

6. Prem K, Liu Y, Russell TW, Adam J Kucharski, Rosalind M Eggo, et al (2020) The effect of control strategies to reduce social mixing on outcomes of the COVID-19 epidemic in Wuhan, China: a modelling study. Lancet Public Health 5(5): e261-e270.

7. De Caro F, Hirschmann TM, Verdonk P (2020) Returning to orthopaedic business as usual after COVID-19: strategies and options. Knee Surg Sports Traumatol Arthrosc 28(6):1699-1704.

8. https://www.ioaindia.org/ Retrieved on 18 August 2020.

9. https://www.boa.ac.uk/resources/boa-guidance for restart - full docfinal 2 pdf.html and nice.org.uk/guidance/ng179/resources/visual summary pdf- 8782806637

10. https://www.efort.org/aiot-joint-statement-covid $\quad-19 \quad$ AIOT recommends global research collaborations, a unification of key recommendations.

11. Position statement on the staged and safe approach to the return of orthopaedic surgery during COVID-19.

12.https://www.cdc.gov/coronavirus/2019-ncov/hcp/clinicpreparedness.html

13. Chang LZ, Wang W, Murphy D, Po Hui JH (2020) Novel Coronavirus and Orthopaedic Surgery: Early Experiences from Singapore. J Bone Joint Surg Am 102(9): 745-749. 
14. Placella G, Salvato D, Delmastro E, Bettinelli G, Salini V (2020) CoViD-19 and ortho and trauma surgery: The Italian experience. Injury 51(6): 1403-1405.

15. Ti LK, Ang LS, Foong TW, Ng BS (2020) What we do when a COVID-19 patient needs an operation: Operating room preparation and guidance. Can J Anaesth 67(6): 756-758.

16. Del Rio C, Malani PN (2020) COVID-19dnew insights on a rapidly changing epidemic. JAMA (ahead of print)

17. Jain VK, Vaishya R (2020) COVID-19 and orthopaedic surgeons: the Indian scenario. Tropical Doctor 50(2): 108-110.

18. Oussedik S, Zagra L, Shin GY, D Apolito R, Haddad FS (2020) Reinstating elective orthopaedic surgery in the age of COVID-19. Bone Joint J 102 $\mathrm{B}(7)$ : 807-810.

\section{Home | Ministry of Health and Family Welfare.}

20. Parvizi J, Gehrke T, Krueger CA, E Chisari M Citak, et al. (2020) Resuming Elective Orthopaedic Surgery During the COVID-19 Pandemic: Guidelines Developed by the International Consensus Group (ICM). J Bone Joint Surg Am 102(14): 1205-1212.

21. Mouton C, Hirschmann MT, Ollivier M, Romain Seil, Jacques Menetrey (2020) COVID-19 - ESSKA guidelines and recommendations for resuming elective surgery. J EXP ORTOP 7(1): 28.

22. Xu S, Li Y (2020) Beware of the second wave of COVID-19. Lancet 395(10233): 1321-1322.

23. Ali I (2020) COVID-19: Are We Ready for the Second Wave? Disaster Med Public Health Prep 7: 1-3. 